\author{
IWONA LEGUTKO-MARSZAŁEK \\ Uniwersytet Gdański
}

\title{
Übersetzung als komplexer kognitiv basierter Prozess
}

\section{Cognitive aspects of the translation}

\begin{abstract}
The following article concentrates on cognitive aspects of the translation. We are interested in course of the translation process in the brain, which is one of the most complicated processes connected with language. It integrates not only the linguistic perception and production, but also code-switching. During the translation process occurs activation of the linguistic information organized in mental lexicon, along with the nonlinguistic knowledge stored in other components of the memory.
\end{abstract}

KEYWORDS: Translation process, lexical access, mental lexicon, cognitive processes.

Der Prozess des Übersetzens soll als eine kreative, strategische und wissensbasierte Leistung einer Person gesehen werden, die auf der Grundlage eines zu übersetzenden Textes in unterschiedlichen Situationen und Handlungszusammenhängen und für unterschiedliche kommunikative Zwecke inhaltliche Äquivalenttexte in einer anderen Sprache erstellt. Übersetzen darf nicht nur als ein interlingualer Transfer bezeichnet werden, in dem ein Text aus einer Sprache in die andere übertragen wird, sondern man sollte auch die subjektive Seite der Planung einer Übersetzung, die Aktivierung kognitiver Ressourcen und den Ablauf mentaler Prozesse berücksichtigen. Übersetzen ist nämlich ein komplizierter, sowohl das sprachliche Wissen in zwei Sprachen als auch das sprachunabhängige, auf individuellen und kulturbedingten Erfahrungen basierende begriffliche Wissen integrierender Prozess. Der Übersetzer selbst soll nicht nur über hervorragende sprachliche und kommunikative Kompetenz in zwei Sprachen, sondern 
auch über Wissen bezüglich der translatorischen Strategien verfügen, d.h. er soll auf kohärenzfördernde referentielle Kontinuität achten, kulturelle Konventionen beachten, texttypologische Varianten berücksichtigen, sprachsystematische Differenzen bewältigen können usw. Der Translator ermöglicht es dem Rezipienten, dass dessen Weltwissen mit der kontextspezifischen Textinformation interagieren und damit der Textsinn und Handlungsziele erkannt werden. Der Inhalt des zu übersetzenden Textes benötigt natürlich auch die erforderlichen Sachkenntnisse. Der Übersetzungsprozess erscheint so als Reaktion auf Vorgaben, Anforderungen und strukturelle Einschränkungen, als Problemlösen und Entwickeln von kognitiven und metakognitiven Strategien. Wichtig dabei ist nicht die Analyse der sprachsystematischen Relationen zwischen dem Ausgangs- und Zieltext, sondern die kognitiven Aspekte des Übersetzungsvorgangs, die dem Entwurf und der Abfassung eines Zieltextes zugrunde liegen. Um Texte korrekt übersetzen zu können, muss man nicht nur über sprachliche und kommunikative, sondern auch über übersetzerische Kompetenz verfügen. Das bedeutet, dass die Übersetzungskompetenz die Zweisprachigkeit voraussetzt, der Bilingualismus dagegen keinesfalls mit dem Übersetzungskönnen gleichzusetzen ist. Sowohl die Entwicklung der Übersetzungskompetenz als auch der Übersetzungsprozess selbst lassen sich aus den kognitiven Grundlagen her erklären. Wir nehmen an, dass die Zweisprachigen, die ihre zweite Sprache zwar nach dem Erwerb der ersten Sprache und in ihrem Kontext erlernt haben, diese aber in solch einem Grad beherrschen, dass sie in jeder Situation sprachlich agieren können und außerdem über hervorragendes metasprachliches Wissen verfügen, zwei separate mentale Lexika für diese Sprachen entwickelt haben (Legutko-Marszałek 2006: 66ff.). Das trifft zum Beispiel auf die Germanistikabsolventen zu, bei denen ihre zweite Sprache im Studium zum Mittel der Kommunikation und Erkenntnisgewinnung wurde, d.h. die Funktion und damit den Rang der ersten Sprache erlangte. Das linguistische, also das fachspezifische Wissen über die Sprache wurde nur mittels der Fremdsprache angeeignet. Im Übersetzungsprozess fungieren die Germanistikabsolventen als Sprachmittler, die ausgangssprachliche Texte aufnehmen, verarbeiten und in die Zielsprache übertragen. Sie sind sogar im Stande, die so genannten "unübersetzbaren“ Einheiten, wie idiomatische Ausdrücke, Sprichwörter, Wörter, die in der anderen Sprache keine Entsprechungen haben (Nulläquivalenz), Wörter, bei denen der Bedeutungsumfang nur teilweise übereinstimmt (partielle Äquivalenz), Kollokationen, Realien (Tatsachen, die mit der Kultur einer Sprachgemeinschaft zusammengewachsen sind), Konnotationen und vor allem Wortspiele von einer Sprache in die andere zu transponieren. Das erfordert eine Fertigkeit, die erlernt und eingeübt werden muss. 
Kognitionswissenschaftlich wird Übersetzen als Problemlösen verstanden; es wird als komplexer mentaler Prozess charakterisiert, in dem die übersetzende Person zuerst einen Ausgangstext dekodieren und verstehen muss, um ihn dann in eine andere Sprache zu übertragen. Der Übersetzungsprozess soll deshalb als ein Sprachverarbeitungsprozess betrachtet und analysiert werden, der sowohl die Sprachrezeption als auch die Sprachproduktion einbezieht. Zwischen der Rezeption in einer Sprache (L1) und der Produktion in einer anderen (L2) ist der kognitive Übersetzungsvorgang zu platzieren. Solche Prozesse laufen selten routinemäßig ab. Die Steuerung der subjektiven Verstehensvorgänge durch bottom-up und top-downProzesse läuft nicht immer automatisch ab. Häufig muss der Translator Szenen bewusst konstruieren, unkontrollierte ZS-Formulierungen überprüfen oder sich vergewissern, dass er das vom Autor vorausgesetzte Weltwissen besitzt. Die automatische, unbewusste Informationsverarbeitung, wie Wahrnehmungsprozesse, reflexhafte Assoziationen von Wörtern, korrekte Satzbildung usw. verläuft tendenziell schnell und parallel. Die kontrollierte, bewusst eingesetzte Informationsverarbeitung verläuft tendenziell langsam und seriell (Gadenne 1996: 107ff.). Bei Sprachproduktionsprozessen sind die bewussten Prozesse grundlegend (Kontrolle über Gedächtnisleistungen und deklaratives Wissen, Fähigkeit zu Selbstkorrekturen etc.). Sie unterliegen der Kontrolle metakognitiver Regulation, also der Kontrolle des Wissens über die eigenen kognitiven Fähigkeiten und Defizite. Dadurch wird der gezielte Einsatz von Strategien in gegebener Situation ermöglicht, die kognitives Verhalten steuern (vgl. Kupsch-Losereit 1997: 212ff.).

Alle kognitiv ablaufenden Informationsverarbeitungsprozesse, darunter auch sprachliche Verarbeitungsprozesse, wie Rezeption und Verstehen, Übersetzen und Produktion, verlangen zumeist das Aktivieren und $\mathrm{Zu}$ sammenwirken vieler verschiedener Gedächtniskomponenten. Bei der übersetzerischen Tätigkeit haben wir mit dynamischen, auf neuronaler Aktivität basierenden Prozessen zu tun, die unser im Gehirn gespeichertes sprachliches, begriffliches und prozedurales Wissen in Anspruch nehmen. Die zentrale Funktion spielt hier das mentale Lexikon, in dem alle lexikalischen Einheiten samt der sprachlichen Informationen gespeichert sind. Im Falle eines Übersetzungsvorgangs haben wir es mindestens mit zwei separaten Lexika $\mathrm{zu}$ tun, weil die übersetzende Person mindestens zweisprachig sein muss, um translatorisch agieren zu können. In Sprachverarbeitungsprozessen wird außer den im mentalen Lexikon gespeicherten sprachlichen Informationen auch das nichtsprachliche begriffliche und prozedurale Wissen abgerufen. Im Prozess der Sprachrezeption wird eine Sequenz von sprachlichen Einheiten in einer Sprache zerlegt, ausgewertet und identifiziert, um einen intendierten Inhalt zu erschließen. Die konzeptuelle Analyse benötigt die Aktivie- 
rung von Wissensrepräsentationen im semantischen Gedächtnis. Im Prozess der Sprachproduktion wird dagegen die entnommene Intention zunächst in sprachliche Strukturen der anderen Sprache umgesetzt und dann ausgedrückt, wobei wiederum auch das prozedurale Gedächtnis aktiviert werden muss. Sowohl der Prozess der Sprachrezeption als auch der Prozess der Sprachproduktion erfordern einen Zugriff auf die in mentalen Lexika der betreffenden Sprachen abgespeicherten sprachlichen Informationen. Mit dem lexikalischen Zugriff haben wir dann zu tun, wenn die zum sensorischen Input im Prozess der Sprachrezeption und zur konzeptuellen Intention im Prozess der Sprachproduktion passenden Einträge, d.h. Zugriffsrepräsentationen auf alle mit einer Sprache zusammenhängenden Informationen, im mentalen Lexikon aktiviert werden (Legutko-Marszałek 2010: 527f.). Auf diese Weise werden in Sprachverarbeitungsprozessen mit einem lexikalischen Eintrag auch folgende sprachbezogene Informationen mitaktiviert:

a) phonetisch-phonologische und graphemische Information (Laut- und Silbenanzahl, die Phonem- und Akzentstruktur, Buchstabenfolge nach den Rechtschreibungsregeln);

b) morphologische Information (entsprechend Deklinations-, Konjugations- und Komparationsflexionsmusterwissen, Derivations- und Kompositionswortbildungs schemata, Wortstrukturwissen bezüglich des Genus, Tempus, Modus, Numerus und der Person und Partizipien);

c) syntaktische Information (die Wortklasse, potentielle Satzgliedstellung, die Satzart, Kombinationsrestriktionen: Argumentstruktur (Valenz), die Subjekt-Prädikat-Objekt-Relation (Rektion, Kongruenz) in Abhängigkeit von Tempus, Genus, Modus);

d) semantische Information (Bezug auf eine begriffliche Wissensrepräsentation (Bedeutung), semantische Merkmalsstruktur, die RelationsArgument-Verbindungen (thematische Rollen), die assoziative Beziehung zu anderen Bedeutungen);

e) pragmatische Information (situativer Kontext, Bezug auf Schemata, Skripts, mentale Modelle).

Im Übersetzungsprozess wird aber abhängig davon, in welche Richtung der Verarbeitungsvorgang verläuft, jeweils auf anderes mentale Lexikon zugegriffen. Das mentale Lexikon bildet zusammen mit dem deklarativen und nicht-deklarativen Wissen (vgl. Squire 1993) Gedächtniskomponenten des Langzeitgedächtnisses. Wie wir bereits angedeutet haben, beginnt der Übersetzungsprozess mit der Sprachrezeption, und genauer mit dem Verstehen eines zu übersetzenden Textes. Verstehen erscheint demnach als ein konstruktiver, sprach-, wissen- und kontextabhängiger Prozess. Sprachverarbeitung bedeutet demnach nicht nur eine Analyse der im Input enthalte- 
nen Informationen, sondern eine aktive Konstruktion einer mentalen Repräsentation, die auch Informationen enthalten kann, die nicht im Input vorhanden sind (vgl. Bransford, Barclay, Franks 1972). Wissensbasierte Inferenzen sind ein integraler Bestandteil der Sprachverarbeitung und verbinden Textinhalte mit dem Wissen über sprachliches Handeln, dem Interaktionswissen sowie Erfahrungs- und Weltwissen, um einen kohärenten und in sich stimmigen Textsinn zu erfassen (Rickheit, Strohner 2003: 566ff.). Verstehen ist also das Resultat kognitiver Prozesse, die aus mentalen Schlussfolgerungen und Problemlösungsoperationen bestehen. Bei der Übersetzung geht es darum, dass der Inhalt möglichst getreu und verständlich aus einer Sprache in die andere übertragen werden soll. Um den Inhalt richtig erraten zu können, muss der $\mathrm{zu}$ übersetzende Text zuerst zergliedert und die Übersetzungseinheiten, seien es die Sätze, Phrasen oder sogar Wörter, müssen analysiert werden. Im Rezeptionsprozess ist die strukturelle Information vorgegeben, sie muss aber richtig dekodiert werden. Das benötigt Aktivierung des deklarativen Gedächtnisses, das grammatisches Wissen bezüglich des sprachlichen Systems der Sprache, in der ein zu übersetzender Text verfasst wurde, enthält. Parallel zur syntaktischen Verarbeitung mit minimaler Verzögerung (vgl. Friederici, Kotz 2003) werden einzelne Wörter prälexikalisch analysiert und erkannt, indem passende lexikalische Einträge im mentalen Lexikon der Ausgangssprache identifiziert werden. Mit dem lexikalischen Zugriff wird aus der Menge der aktivierten Lexikoneinheiten dasjenige Wort ausgewählt, das am besten mit dem Input übereinstimmt. Nach der Auswahl eines entsprechenden Eintrags werden andere mit ihm assoziativ verbundene Einträge deaktiviert. Die mit den Lexikoneinträgen aktivierten phonetisch-phonologischen, morphologischen, semantischen, syntaktischen und pragmatischen Informationen werden im Rahmen der gesamten sprachlichen Struktur integriert. Durch die Aktivierung der den lexikalischen Einheiten entsprechenden begrifflichen Wissensrepräsentationen wird schließlich die Bedeutungsstruktur eines Satzes erschlossen. Einfluss auf die Interpretation sprachlicher Übersetzungseinheiten nimmt auch der situative Kontext, der die konzeptuelle Struktur modifiziert und beispielsweise mehrdeutige Bedeutungen disambiguiert. Der Rezipient eines Textes baut Relationen zwischen den im Satz oder Text genannten Einheiten und Ereignissen auf, indem er auf sein im Langzeitgedächtnis gespeichertes Wissen zurückgreift. Dieser Vorgang verlangt von dem Übersetzer ein allgemeines, auf Erfahrungen basierendes, kulturbezogenes oder sogar fachspezifisches Wissen.

Nachdem die konzeptuelle Struktur erfasst worden ist, kann mit der Übersetzung in die andere Sprache begonnen werden. In einem Sprachproduktionsprozess aktiviert der Mensch normalerweise zunächst Teile seines 
gespeicherten sprachunabhängigen konzeptuellen Wissens, um eine kognitive Repräsentation des intendierten Äußerungsinhalts zu erstellen. Anders als im gewöhnlichen Sprachproduktionsprozess muss im Übersetzungsprozess keine konzeptuelle Struktur erzeugt werden, weil die im Ausgangstext schon vorgegeben ist. Im Übersetzungsprozess muss diese Inhaltsstruktur dem Text in einer Sprache entnommen und mittels des anderen sprachlichen Systems wiedergegeben werden. Deshalb wird von einer Sprache auf die andere umgeschaltet, um mit strukturell-systematischen Mitteln der Zielsprache einen äquivalenten Text zu produzieren. Dieser Vorgang benötigt eine Aktivierung des mentalen Lexikons der Sprache, in die ein Text übersetzt werden soll. Folglich werden die lexikalischen Einheiten selektiert, die den begrifflichen Wissensrepräsentationen entsprechen. Der Zugriff auf die passenden lexikalischen Einträge der Zielsprache kann auf zweierlei Wegen erfolgen: entweder über Konzepte oder direkt, d.h. ohne Bezug auf das Konzept. An dieser Stelle soll auf den Unterschied zwischen sprachlichen Bedeutungen und nichtsprachlichen Konzepten hingewiesen werden (LegutkoMarszałek 2007: 163ff.). Begriffe sind prinzipiell sprachunabhängig und frei von jeglichen Sprachbegrenzungen. Mit dem Erwerb einer Sprache werden sie jedoch definiert und ihnen wird eine sprachliche Wortform konventionellerweise zugeordnet, d.h. sie werden lexikalisiert. Von diesem Moment an unterliegen sie nicht nur den Erfordernissen der Kognition, sondern auch denen der Kommunikation und der grammatischen Strukturbildung. Die sprachlichen Bedeutungen müssen also sozial akzeptiert, klar definiert, relativ stabil und objektiv sein und außerdem mit anderen lexikalisierten Begriffen strukturelle Beziehungen eingehen. Die überindividuellen sprachgebundenen Bedeutungen sind von den reinen Konzepten zu unterscheiden und werden als Operationen aufgefasst, die darin bestehen, der jeweiligen Wortform entsprechende konzeptuelle Schemata zu suchen, diese zu aktivieren und gemäß dem aktuellen sprachlichen und nichtsprachlichen Kontext zu konkretisieren, d.h. bestimmte Variablen durch dafür in Frage kommende Konstanten zu belegen (Scherfer 1997: 198). In einem Übersetzungsprozess wird durch die Identifizierung eines lexikalischen Eintrags im mentalen Lexikon über sprachliche Bedeutung auch die begriffliche Wissensrepräsentation aktiviert, die dann durch eine anderssprachige Bedeutung ausgedrückt werden muss. Es kann aber passieren, dass man auf der sprachlichen Ebene, d.h. ohne Bezug auf das außersprachliche Konzept einen entsprechenden Eintrag auffinden kann. Als Beispiel könnte hier die Übersetzung von Fachtexten dienen. In dieser Situation kann man die sprachliche Bedeutung verstehen und sie in der anderen Sprache richtig wiedergeben, ohne über den Begriff zu verfügen, z.B. Schelf - szelf, Erosion - erozja, tektonisch tektoniczny usw. Das ist möglich, weil, obwohl die mentalen Lexika der bei- 
den Sprachen getrennt sind, sie doch durch die sprachlichen Äquivalentverbindungen miteinander interagieren. Mit dem Zugriff auf einen lexikalischen Eintrag werden nicht nur Informationen über phonetisch-phonologische Formen, morphologische Strukturen, semantische Repräsentationen und syntaktische Regularitäten, die auf die intrinsische Struktur des mentalen Lexikons hinweisen, abgerufen. Auch die mit der lexikalischen Einheit assoziativ verbundenen Einträge, wie die über relationale Vernetzungen nahen Bedeutungen, die phonologisch ähnlichen Wörter oder die Äquivalente der angeeigneten weiteren Sprache, werden aktiviert. Normalerweise werden die der konzeptuellen Struktur entsprechenden Einträge im mentalen Lexikon der Zielsprache ausgewählt und die übrigen deaktiviert. Während der Lexikalisierung wird unter Berücksichtigung aller mit den lexikalischen Einheiten automatisch aktivierten sprachlichen Informationen die syntaktische Struktur erstellt, die den grammatischen Restriktionen der Sprache entspricht, in die übersetzt wird. Es werden im mentalen Lexikon der Zielsprache aber nicht immer nur Äquivalente selektiert. Der situative Kontext, die konzeptuelle und grammatische Struktur als auch Inferenzen beeinflussen in großem Maße die Auswahl der zielsprachigen Einheiten. Im Sprachrezeptionsprozess werden die potentiellen Äquivalente in der Zielsprache aktiviert, weil die mentalen Lexika beider Sprachen vernetzt sind. Besonders bei einfachen Satzkonstruktionen -sprachsystematische Einstimmigkeit (Subjekt-Prädikat-Objekt-Relation) vorausgesetzt- fällt das Übersetzen sehr leicht, indem man aktivierte sprachliche Äquivalente in die syntaktische Struktur der Zielsprache einsetzt, wie z.B. Die Mutter kauft dem Kind das Buch. Matka kupuje dziecku książkę. Sogar in Sätzen, in denen wir mit keiner strukturellen Übereinstimmung zu tun haben, kann man sich der aktivierten Äquivalente bedienen, wie z.B. Das Mädchen ist seinem Freund begegnet. Dziewczynka spotkała przyjaciela. Mit solchen Fällen haben wir jedoch bei der Übersetzung sehr selten zu tun. Viel öfter müssen die automatisch aktivierten Äquivalente revidiert und durch andere, den begrifflichen Wissensrepräsentationen entsprechende Einheiten ersetzt werden, z.B. (1) Der Beamte folgte dem Rat des Bürgermeisters. Urzędnik postuchat rady burmistrza. (2) Sie hat mich mit ihrem Blick durchbohrt. Przeszyła mnie swoim wzrokiem. (3) Dem Ingenieur ist ein schwerer Fehler unterlaufen. Inżynier popetnił duży btad. (4) Ale jesteś wysoki. Du bist aber groß. (9) Der Student hat in der Prüfung versagt. Student nie zdat egzaminu. Der Übersetzungsvorgang wird noch komplizierter, wenn wir mit komplexen Satz- und Nebensatzkonstruktionen zu tun haben, wie in diesem Artikel, wobei wir uns nicht mehr auf einzelne Konzepte, sondern auf die kognitive Repräsentation des intendierten Inhalts beziehen müssen, z.B. Es ist lebensbedrohlich, von einer solchen Schlange gebissen zu werden. Ugryzienie przez taka żmije zagraża życiu (in der deutschen Sprache wird die 
Infinitivkonstruktion gewählt und in der polnischen Sprache wird der Teil nominalisiert). Die Übersetzung aus einer Sprache in die andere ist nur deshalb möglich, weil wir die Fähigkeit besitzen, von den sprachlichen Systemen zu abstrahieren und auf der sprachunabhängigen konzeptuellen Ebene Informationen zu verarbeiten.

Zusammenfassend kann man feststellen, dass im Übersetzungsprozess zuerst die einzelnen sprachlichen Einheiten im mentalen Lexikon der Ausgangssprache identifiziert und selektiert werden, wobei die sprachlichen Bedeutungen ihre begriffliche Wissensrepräsentationen aktivieren, und auf diese Weise die konzeptuelle Struktur einer formulierten Übersetzungseinheit erstellt wird. Zu diesen Begriffen müssen folgend im mentalen Lexikon der Zielsprache die passenden Äquivalente ausgewählt werden. Im Rezeptionsprozess werden auch die assoziativen Verbindungen mit ausgangsprachlichen Einheiten eingehenden zielsprachigen Äquivalente voraktiviert und, wenn sie die konzeptuelle Struktur nicht verletzen, akzeptiert. Bei Nichtübereinstimmung werden sie revidiert und durch andere lexikalische Einheiten ersetzt, die der konzeptuellen Struktur entsprechen. Manchmal muss sogar die Bedeutungsstruktur eines Satzes in der Ausgangssprache komplett geändert und in der Zielsprache durch ganz andere ersetzt werden, wie es bei idiomatischen Ausdrücken, festen Redewendungen, Wortspielen oder Sprichwörtern der Fall ist. Schließlich werden die ausgewählten sprachlichen Einheiten zur motorischen Ausführung bereitgestellt. Im Übersetzungsprozess wird also dem in einer Sprache verfassten Satz die konzeptuelle Struktur entnommen und mit Mitteln einer anderen Sprache wiedergegeben. Mit den dargestellten Schritten wird dann der ganze Text übersetzt. Schwierigkeiten bereitet die richtige Erfassung und Wiedergabe des Sinns und der Intention eines Textes und das wiederum kann nur dann geschehen, wenn die sprachlichen Texte richtig verstanden werden, d.h. die konzeptuelle Struktur korrekt entschlüsselt wird.

Wie wir sehen, gehört der Übersetzungsvorgang zu den kompliziertesten Sprachverarbeitungsprozessen, der nicht nur die Sprachrezeption und Sprachproduktion in sich integriert, sondern auch den Sprachwechsel einbeziehen muss. Dabei wird nicht nur auf die mentalen Lexika beider Sprachen, wo die lexikalischen Einheiten samt allen sprachlichen Informationen gespeichert sind, zugegriffen, sondern es werden auch andere Gedächtniskomponenten aktiviert, wo das Wissen bezüglich des sprachlichen Systems und der Anwendung von grammatischen Regeln gespeichert ist. Der ganze Prozess muss natürlich auch überwacht und kontrolliert werden, so dass die sprachlichen Unzulänglichkeiten auf der Stelle korrigiert werden. Der Komplexität dieser in unserem Gehirn ablaufenden Prozesse sind wir uns nicht immer bewusst, weil sie sehr schnell und zum Teil automatisiert ablaufen, 
wie die Sprachproduktion und -rezeption oder perfekt eingeübt werden, so dass sie keine größeren Schwierigkeiten bereiten, wie der Übersetzungsvorgang. Nur wenn wir auf Probleme stoßen, machen wir uns Gedanken darüber. Die Übersetzungskompetenz erfordert aber nicht nur Erlernung und Einübung einer Fertigkeit, sondern auch einer gewissen Fähigkeit, zwischen zwei Sprachsystemen zu balancieren. Beweis dafür sind zahlreiche misslungene Übersetzungen.

\section{LITERATURVERZEICHNIS}

Bransford, J.D., Barclay, J.R., Franks, J.J., 1972. Sentence memory: A constructive vs. interactive approach. In: Cognitive Psychology 3, 193-209.

Friederici, A.D., Kotz, S.A., 2003. The brain basis of syntactic processes: functional imaging and lesion studies. In: Neuroimage 20, Suppl. 1, 8-17.

Gadenne, V., 1996. Bewusstsein, Kognition und Gehirn. Einführung in die Psychologie des Bewusstseins. Bern: Verlag Hans Huber.

Kupsch-Losereit, S., 1997. Übersetzen: ein integrativ-konstruktiver Verstehens- und Produktionsprozess. In: Drescher, H.W. (Hrsg.). Transfer. Übersetzen - Dolmetschen - Interkulturalität. Frankfurt: Peter Lang.

Legutko-Marszałek, I., 2006. Zum Status eines mentalen Lexikons bei einem bilingualen Sprecher. In: Glottodidactica XXXII. An International Journal of Applied Linguistics, 59-71.

Legutko-Marszałek, I., 2007. Die Unterscheidung zwischen Bedeutungen und Begriffen in Anbetracht des Bilingualismus. In: Studia Germanica Gedanensia, 163-168.

Legutko-Marszałek, I., 2010. Der Zugriff auf lexikalische Einheiten und die Aktivierung von sprachlichen Informationen im Prozess der Sprachrezeption und Sprachproduktion bei Bilingualen. In: Studia Niemcoznawcze XLV, 525-535.

Rickheit, G., Strohner, H., 2003. Inferenzen. In: Rickheit, G., Herrmann Th., Deutsch, W., (Hrsg.) Psycholinguistik. Ein internationales Handbuch. Berlin - New York: Walter de Gruyter.

Scherfer, P., 1997. Überlegungen zu einer Theorie des Vokabellernens und -lehrens. In: Börner, W., Vogel, K. (Hrsg.). Kognitive Linguistik und Fremdsprachenerwerb. Tübingen: Gunter Narr Verlag.

Squire, L., 1993. The organization of declarative and non-declarative memory. In: Ono T., Squire, L., Raichle, M.F., Perrett D., Fukuda, J. (Hrsg.). Brain mechanism of perception and memory: From neuron to behavior. New York: McGraw-Hill. 
